EPJ Web of Conferences 73, 02018 (2014)

DOI: $10.1051 /$ epjconf/20147302018

(C) Owned by the authors, published by EDP Sciences, 2014

\title{
Studies of partonic transverse momentum and spin structure of the nucleon
}

\author{
M. Contalbrigo ${ }^{\mathrm{a}}$ \\ INFN - Sezione di Ferrara, 44122 Ferrara, Italy
}

\begin{abstract}
The investigation of the partonic degrees of freedom beyond collinear approximation (3D description) has been gained increasing interest in the last decade. The Thomas Jefferson National Laboratory, after the CEBAF upgrade to $12 \mathrm{GeV}$, will become the most complete facility for the investigation of the hadron structure in the valence region by scattering of polarized electron off various polarized nucleon targets. A compendium of the planned experiments is here presented.
\end{abstract}

\section{The 3D structure of nucleons}

Since decades, few questions challenge the interpretation of hadron structure and phenomena in Hadronic Physics in terms of perturbative QCD. Among the most intriguing, there is the spin budget of the nucleon, where there are missing contributions not quantified yet, and the surprising single-spin asymmetries, which do not vanish as expected with increasing energy. The questions above relate to one of the fundamental degree of freedom of the elementary particles, the spin, and its correlation with the particle motion (transverse momentum) of the partons.

Most of our present understanding of the internal structure of nucleons derives from inclusive deep-inelastic-scattering (DIS) experiments performed over the past four decades in different kinematic regimes at fixed-target experiments and collider machines. Based on the large amount of precise data provided by these experiments we have reached a good knowledge of the longitudinal-momentum and longitudinal-spin distributions of quarks in the nucleon, where "longitudinal" refers to the direction of the exchanged virtual boson (the hard probe). A complete collinear description of the nucleon structure at leading order in an expansion in M/Q (twist expansion), where $\mathrm{Q}$ is the photon virtuality and $\mathrm{M}$ the nucleon mass, requires the knowledge of three fundamental parton distributions: the momentum distribution $f_{1}(x)$, the helicity distribution $g_{1}(x)$, and the presently poorly known transversity distribution $h_{1}(x)$. Here $x$ denotes the longitudinal momentum fraction carried by the partons and the scale $\left(Q^{2}\right)$ dependence has been neglected for simplicity. Although transversity is related to the tensor charge of the nucleon [1], it has for long remained unmeasured due to its chiral-odd nature, which prevents its measurement in inclusive DIS.

In the recent years, new parton distributions have been introduced to describe the rich complexity of the hadron structure, taking into account the parton transverse degrees of freedom and moving toward

\footnotetext{
ae-mail: contalbrigo@fe.infn.it
}

This is an Open Access article distributed under the terms of the Creative Commons Attribution License 4.0, which permits unrestricted use, distribution, and reproduction in any medium, provided the original work is properly cited. 
the achievement of a 3D comprehension of the parton dynamics. At the same time new channels of investigation have been gained importance as the study of semi-inclusive deep-inelastic scattering (SIDIS) where at least one final state hadron is detected in coincidence with the scattered lepton. The SIDIS cross-section provides access to a rich phenomenology as can be described in terms of convolutions of parton distribution and fragmentation functions. Besides allowing to access transversity in conjunction with the Collins fragmentation function, SIDIS experiments open the way to the extraction of transverse-momentum-dependent (TMD) parton distributions [2]. Describing correlations between the quark or the nucleon polarization and the quark transverse momentum, i.e. spin-orbit correlations, the TMD distribution functions encode information on the 3-dimensional parton dynamics and are increasingly gaining theoretical and experimental interest. There are five independent leadingtwist quark TMDs in addition to the parton distributions above introduced. Their existence requires nonzero orbital angular momentum as they are related to the wave function overlap of Fock states of the nucleon with different angular momentum. The chiral-even distributions $f_{1 T}^{\perp}$ and $g_{1 T}$ are the imaginary parts and the chiral-odd $h_{1}^{\perp}$ and $h_{1 L}^{\perp}$ are the real parts of the interference terms between $S$ and $P$ wave components. The chiral-odd $h_{1 T}^{\perp}$ function is sensitive to the $D$-wave component. In particular, the TMDs $f_{1 T}^{\perp}$ and $h_{1}^{\perp}$ are known as the Sivers [3] and Boer-Mulders [4] functions. They require a non-trivial gauge link and therefore exhibit a peculiar process dependence: a sign change is expected moving from SIDIS to Drell-Yan processes.

\section{The Thomas Jefferson National Laboratory (JLab)}

Several interesting results related to the spin structure of the nucleon have been achieved during the almost 15 years of the JLab continuous electron beam accelerator facility (CEBAF) activity at $6 \mathrm{GeV}$. Among those are the evidence that the electric and magnetic moments of the proton does not scale as suggested by the historical Rosembluth separation [5] (likely because of underestimated radiative corrections), one of the first observations of a non-zero DVCS asymmetry [6], the hint of a different transverse momentum distribution for up and down quarks [7], the first TMD measurements with a transversely polarized ${ }^{3} \mathrm{He}$ target accessing the neutron distribution functions [8].

The 3D studies of the spin nucleon structure (in terms of TMDs) will greatly benefit from the higher energy and luminosity available at $11 \mathrm{GeV}$, after the completion of the ongoing machine upgrade. The higher beam energy is ideal to efficiently map the quark valence region in the few $\mathrm{GeV}$ hard-scale regime, covering a poorly explored region at large $x$ which is crucial for the understanding of the spin dependent processes. The precision mapping in multi-dimensions will not only allow for the extraction of the new distributions, but also for a careful study of the reaction mechanisms and related issues, such as the factorization, the separation/mixing in current-target fragmentation processes and the higher-twist effects. The SIDIS measurements will be complemented by the simultaneous measurements of hard exclusive reactions (accessing the so-called generalized parton distributions) and elastic scattering to extract the proton and neutron form factors at high $Q^{2}$. A comprehensive program is in preparation to exploit the complementary of the different JLab experimental halls in the study the new quark distribution functions, as described in the following.

Hall $\mathbf{C}$ and the factorization tests: the SIDIS program in Hall $\mathrm{C}$ is focused on precision cross section measurements with the anticipated excellent systematic understanding of the HMS-SHMS highmomentum magnetic spectrometer pair. The well-shielded detector systems, the rigid connection to a sturdy pivot, the well-reproducible magnetic properties, and the access to the highest-luminosity data taking makes the HMS-SHMS setup optimal for a program of longitudinal to transverse cross-section separation [9] and ratios of charged-meson cross sections [10].

Hall B and the comprehensive exploration: the CLAS12 large acceptance detector will provide a complete set of measurements extending from target to current fragmentation and to exclusive processes, using both unpolarized and polarized hydrogen and deuteron targets. 

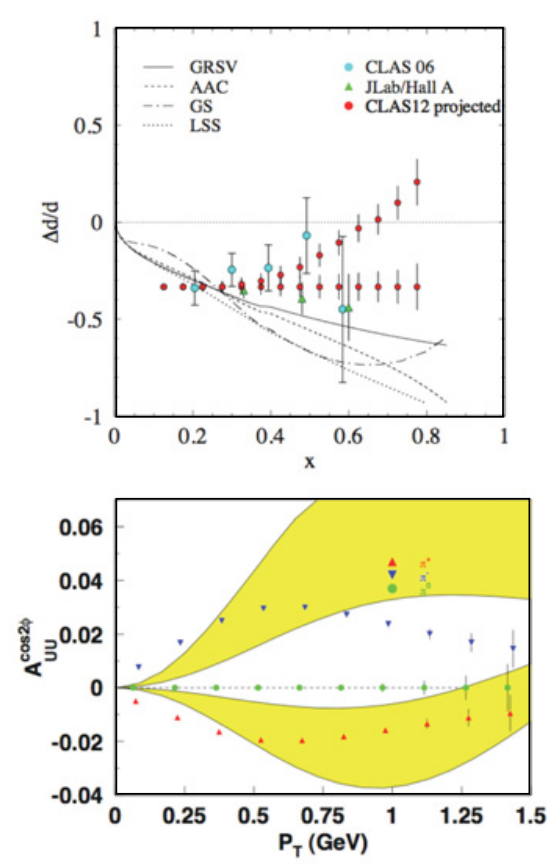

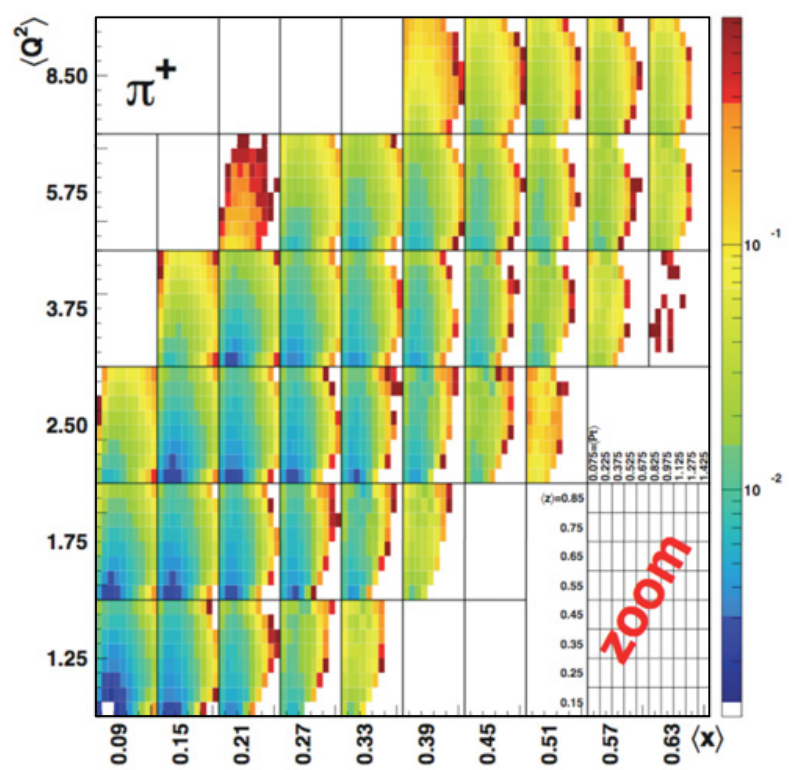

Figure 1. Examples of TMDs measurements planned at $12 \mathrm{GeV}$. Top-left: expected results for $(\Delta d+\Delta \bar{d}) /(d+\bar{d})$ helicity distribution ${ }^{17}$. The central values of the data are following two arbitrary curves to mimic the two categories of predictions (namely $\Delta d / d$ stays negative or tends to 1 ). Bottom-left: expected statistical precision on the BoerMulders asymmetry ${ }^{15}$ for pions as a function of $p_{T}$ for $Q^{2}>2 \mathrm{GeV}^{2}$. The yellow band between the two curves indicates the range of two model predictions. Right: kinematic coverage of the 4-dimensional $\pi^{+}$yield in $Q^{2}, x, z$, and $p_{T}$. The color code shows the projected uncertainties in each bin ${ }^{20}$.

The wide ranges in $Q^{2}$ and $p_{T}$ would allow to separate contributions from leading and sub-leading effects and study all relevant 3D partonic distributions while at the same time gather information on the fragmentation process thanks to the multi-dimensional analyses. Special attention is given to the flavor decomposition of TMDs, thanks to the different nucleon targets and the excellent hadron identification in the final state achieved by time-of-flight and ring-imaging Cherenkov detection techniques.

Precise data from unpolarized liquid hydrogen and deuterium targets will be important to study the role of the sea partons, of the up-to-date poorly know transverse momentum dependence of the quark momentum distribution and of the Boer-Mulders effect with pions [11] and kaons [12]. The goal of the experiments with longitudinally polarized $\mathrm{NH}_{3}$ and $\mathrm{ND}_{3}$ targets in conjunction with the high beam longitudinal polarization is the study of quark helicity with unprecedented precision, in particular at large $x$ where powerful discrimination among models and potential implications for the quark angular momentum reside [13], and the study of azimuthal asymmetries originated by spin-orbit parton correlations by detecting pions [14] and kaons [15] in the final state. The much anticipated use of the HD-ice, a novel type of target with only polarizable material except for a minimum amount of Aluminum for cooling, has the goal of minimize the nuclear effects. Its use with transverse polarization is foreseen for precise extraction of the Sivers and transversity functions with flavor sensitivity in the SIDIS single-hadron [16] and double-hadron [17] production channels.

Hall A and the precision frontier: the $12 \mathrm{GeV}$ SIDIS two-stages program in Hall A is focused on high-statistics measurements of the neutron structure by using the world-leading polarized ${ }^{3} \mathrm{He}$ target. The first stage foresees the use of the Super-Bigbite spectrometer (SBS) for detecting mesons and the BigBite (BB) spectrometer for detecting scattered electrons with a transversely-polarized ${ }^{3} \mathrm{He}$ target will 
allow to measure SIDIS reactions on both charged pions and charged kaons [18]. The apparatus is able to sustain very high luminosities, up to $10^{38} \mathrm{~cm}^{-2} \mathrm{~s}^{-1}$ but offer a limited acceptance. The spectrometers will be set at large angles in order to reach large- $\mathrm{Q}^{2}$ and high $x$, while avoiding the excessive background at small angles.

The second stage foresees the use of a barrel detector embedded in a solenoid (SoLID) downstream of the target, arranged for forward angle (6.6-22 degrees) coverage and charged-pion identification capability. Such a detector should be able to eventually conjugate high luminosity (up to $10^{36} \mathrm{~cm}^{-2} \mathrm{~s}^{-1}$ ) together with large acceptance, providing the ultimate precision limit in the multi-dimensional TMDs exploration at JLab, although with a limited flavor sensitivity because of the lack of kaon identification.

The use of transversely polarized $\mathrm{NH}_{3}$ and ${ }^{3} \mathrm{He}$ targets is planned to study the TMD distributions of proton [19] and neutron [20], respectively, and eventually disentangle the up and down quark distributions. The use of longitudinally polarized ${ }^{3} \mathrm{He}$ target [21], will allow measurements related to the longitudinal-transverse spin-orbit interference.

\section{References}

[1] V. Barone, A. Drago and P.G. Ratcliffe, Phys. Rep. 359, 1 (2002); V. Barone, F. Bradamante, A. Martin, Prog. Part. Nucl. Phys. 65, 267 (2010)

[2] A. Bacchetta et al., JHEP 02, 093 (2007); P.J. Mulders and R.D. Tangerman, Nucl. Phys. B 461, 197 (1996); erratumibid. B 484, 538 (1997)

[3] D.W. Sivers, Phys. Rev. D 41, 83 (1990)

[4] D. Boer and P.J. Mulders, Phys. Rev. D 57, 5780 (1998)

[5] M.K. Jones et al., Phys. Rev. Lett. 84, 1398 (2000); O. Gayou et al., Phys. Rev. C 64, 038292 (2001); O. Gayou et al., Phys. Rev. Lett. 88, 092301 (2002)

[6] S. Stepanyan et al., Phys. Rev. Lett. 87, 182002 (2001)

[7] H. Mkrtchyan et al., Phys. Lett. B 665, 20 (2008)

[8] X. Qian et al., Phys. Rev. Lett. 107072003 (2011); J. Huang et al., Phys. Rev. Lett. 108, 052001 (2012)

[9] JLab experiment E12-06-104, R. Ent et al. (2006)

[10] JLab experiment E12-09-017, R. Ent et al. (2009)

[11] JLab experiment E12-06-112, H. Avakian et al. (2006)

[12] JLab experiment E12-09-008, H. Avakian et al. (2009)

[13] JLab experiment E12-09-007, K. Hafidi et al. (2009)

[14] JLab experiment E12-07-107, P. Rossi et al. (2007)

[15] JLab experiment E12-09-009, H. Avakian et al. (2009)

[16] JLab experiment C12-11-111, M. Contalbrigo et al. (2011)

[17] JLab experiment C12-12-109, H. Avakian et al. (2012)

[18] JLab experiment E12-09-018, B. Wojtsekhowski et al. (2009)

[19] JLab experiment E12-10-006, H. Gao et al. (2010)

[20] JLab experiment E12-11-108, H. Gao et al. (2011)

[21] JLab experiment E12-11-007, J. Huang et al. (2011) 